Reprod. Nutr. Dévelop., 1980, 20 (5 A), 1429-1437.

\title{
Evolution de la teneur en androsténone du tissu adipeux dorsal chez le porc mâle entier de tỵpe Large White : variations selon les conditions d'élevage
}

\author{
par M. BONNEAU, B. DESMOULIN
}

avec la collaboration technique de G. CONSEIL, J.-C. HULIN, R. CHALIER, P. PEINIAU

Station de Recherches sur l'Elevage des Porcs, I. N. R. A., 78350 jouy-en-josas.

Summary. Backfat androstenone content in entire male pigs of the Large-White breed : Variations according to social conditions during rearing.

The effect of social conditions during rearing on variations in backfat androstenone content according to liveweight was studied in two experiments. In experiment 1, 34 entire male pigs were compared according to a $2 \times 2$ factorial design : they were reared (i) in either individual or collective pens and (ii) with or without visual and olfactive confact with gilts of the same age.

In experiment 2, 20 entire male pigs were reared in contact with gilts in either individual or collective pens. Androstenone was radioimmunoassayed twice in each animal, once in a backfat biopsy at $80 \mathrm{~kg}$ liveweight, and again in a backfat sample taken from the carcass. The pigs were slaughtered at $95 \mathrm{~kg}$ liveweight in experiment 1 and at $107 \mathrm{~kg}$ in experiment 2.

At $80 \mathrm{~kg}$ liveweight (approximately 150 days of age), mean androstenone content was low $(0.31 \mu \mathrm{g} / \mathrm{g}$ in experiment 1 and $0.41 \mu \mathrm{g} / \mathrm{g}$ in experiment 2) but wide variability was already evident (fig. 1). When the animals were slaughtered at an early stage $(95 \mathrm{~kg}$, 167 days in experiment 1 ), the mean androstenone content was only $0.55 \mu \mathrm{g} / \mathrm{g}$ and showed high variability $(0.23$ to $2.55 \mu \mathrm{g} / \mathrm{g})$. When the pigs were slaughtered at a later stage $(107 \mathrm{~kg}$, 189 days in experiment 2), the mean androstenone content was higher $(1.27 \mu \mathrm{g} / \mathrm{g})$ and variability ranged between 0.21 and $5.13 \mu \mathrm{g} / \mathrm{g}$. Individual variation according to liveweight revealed two groups of animals :

- 32 (experiment 1) and 40 p. 100 (experiment 2) of male pigs showed no variation beween the two stages,

- the other animals (68 and 60 p. 100) evidenced increased backfat androstenone content. However, the rate of increase showed wide individual variation.

At $80 \mathrm{~kg}$ liveweight, backfat androstenone content was not affected by the social conditions during rearing. At $95 \mathrm{~kg}$ (slaughter weight in experiment 1), the mean adrostenone content was unaffected by the social conditions : however, the proportion of animals exhibiting no increase in androstenone content between 80 and $95 \mathrm{~kg}$ was significantly higher in those reared with no contact with females (fig. 2). At $107 \mathrm{~kg}$ (slaughter weight in experiment 2), the mean androstenone content was significantly higher when the animals were reared in collective pens (table 3 ).

Neither experiment showed any relationship between backfat androstenone content and production parameters (feed intake, growth performance, feed efficiency). The coefficients of correlation between androstenone and carcass characteristics were not signifi- 
cant in experiment 1 . In experiment 2, where the androstenone content was higher, a positive significant relationship was observed between the androstenone and the parameters closely related to the proportion of carcass muscle (table 4).

\section{Introduction.}

Le principal composé responsable des odeurs, dites « sexuelles », qui se manifestent lors de la cuisson des viandes de certains porcs mâles entiers a été isolé par Patterson (1968) ; il s'agit de l'androsténone ( $5 \alpha$-androst-16-ène-3-one). Ce stéroïde à odeur urinaire prononcée, est synthétisé dans le tissu interstitiel du testicule puis stocké dans le tissu adipeux. La concentration en androsténone de ce tissu constitue ainsi une première estimation quantitative des défauts dus aux odeurs sexuelles des viandes.

La teneur en androsténone du tissu adipeux dépend du niveau de production testiculaire de ce stéroïde, lui-même lié au degré de maturité sexuelle des animaux, variable en fonction de l'âge et des conditions d'élevage. Ainsi, chez des porcs de type Landrace Allemand ou Hampshire (Claus, 1975) et chez des animaux de type Landrace Norvégien (Andresen, 1976), la concentration en ce stéroïde dans le tissu adipeux dorsal varie fortement entre 100 et 250 jours d'âge. Dans les conditions de la production, les porcs sont abattus à un poids commercial constant, donc à un âge variable. Or on ne connaît pas l'évolution de la teneur en androsténone avant le stade d'abattage chez le porc mâle de type Large White. Par ailleurs, l'isolement ou l'élevage dés jeunes verrats en l'absence de femelles entraînerait un retard dans leur développement sexuel (Hemsworth et al., 1977a, b, 1978 ; Thomas ef al., 1979). On peut penser que ces conditions d'élevage pourraient également influencer les niveaux de stockage d'androsténone dans les tissus gras. Aussi avons-nous étudié l'évolution de la teneur en androsténone du tissu adipeux dorsal entre le stade $80 \mathrm{~kg}$ de poids vif et l'abattage chez le jeune porc mâle entier de type Large White placé dans différentes conditions d'environnement social.

\section{Matériel et méthode.}

1. Schéma expérimental. - Deux expériences consécutives ont été réalisées en utilisant des porcs mâles de type Large White.

Au cours de la première expérience, 34 porcelets mâles entiers pesant en moyenne $28 \mathrm{~kg}$ à 80 jours ont été répartis entre 4 lots selon un schéma factoriel $2 \times 2$ : deux modes de logement (loges individuelles ou loges collectives de 6 animaux) et deux modes d'élevage (présence ou absence de femelles) ont éfé comparés. Les mâles élevés en présence de femelles, étaient en contact visuel, auditif et olfactif avec une ou plusieurs femelles impubères placée(s) dans une loge adjacente à la leur.

Au cours de la seconde expérience, 20 porcelets mâles entiers pesant en moyenne $27 \mathrm{~kg}$ à 74 jours ont été répartịs en 2 lots permettant de comparer deux modes de logement (en loges individuelles ou collectives). Les animaux étaient tous élevés «i en présence de femelles». 
Dans les deux expériences, un nombre égal de porcs mâles castrés ont été élevés dans les mêmes conditions.

Les porcs sont nourris à volonté avec des régimes à base de maïs et de tourteau de soja contenant $3370 \mathrm{kcal}$ d'énergie digestible ef $17 \mathrm{p}$. 100 de matières azotées en période de croissance (de 27 à $50 \mathrm{~kg}$ ) puis $3340 \mathrm{Kcal}$ d'énergie digestible ef 14 p. 100 de matières azotées en finition (de $50 \mathrm{~kg}$ à l'abattage).

2. Stades d'abattage et mesures de la composifion corporelle. - Les porcs sont abattus à un stade léger ( $95 \mathrm{~kg}$ de poids vif) dans la première expérience et à un stade plus lourd (107 kg) au cours de la seconde. Dès la saignée les testicules sont prélevés et pesés. La longueur de la carcasse et les épaisseurs de lard dorsal sont mesurées sur la carcasse chaude. Après la découpe parisienne de la demi-carcasse froide, le poids ef la densité des fractions de découpe sont déterminés selon Desmoulin (1970). En outre, la surface du muscle long dorsal est mesurée sur une section transversale pratiquée entre les $13^{\mathrm{e}}$ et $14^{\mathrm{e}}$ vertèbres dorsales.

3. Teneurs en androsténone des graisses. - Les concentrations en androsténone du tissu adipeux dorsal des porcs mâles entiers sont déterminées à deux reprises pour chaque animal : une première fois à $80 \mathrm{~kg}$ de poids vif sur une biopsie de tissu gras, une seconde fois à l'abattage. Le dosage radioimmunologique de ce stéroïde a été réalisé selon la méthode mise au point chez le porc par Claus (1974) et les résultats sont exprimés en microgrammes $(\mu \mathrm{g})$ d'androsténone par gramme de lipides.

\section{Résultats.}

1. Performances de croissance et composition corporelle (tabl. 1 et 2). - Les porcs étant alimentés à volonté, le gain de poids vif journalier ne diffère pas significative-

TABLEAU 1

Performances zootechniques

\begin{tabular}{|c|c|c|c|c|c|c|}
\hline & \multicolumn{3}{|c|}{ Première expérience } & \multicolumn{3}{|c|}{ Deuxième expérience } \\
\hline & Entiers & & Castrats & Entiers & & Castrats \\
\hline \multicolumn{7}{|l|}{$\begin{array}{l}\text { Quantité d'aliment consommée } \\
(\mathrm{kg} / \mathrm{j})\end{array}$} \\
\hline $27-50 \mathrm{~kg} \ldots \ldots \ldots \ldots$ & 1,95 & $* *$ & 2,11 & 1,92 & $*$ & 2,03 \\
\hline $50 \mathrm{~kg}$-abattage $\ldots \ldots \ldots \ldots \ldots$ & 2.70 & $* *$ & 2,93 & 2,54 & * & 2,78 \\
\hline $27 \mathrm{~kg}$-abattage ... & 2,38 & $* * *$ & 2,62 & 2,32 & $* * *$ & 2,53 \\
\hline \multicolumn{7}{|l|}{ Gain de poids vif (kg/i) } \\
\hline $27-50 \mathrm{~kg} \ldots \ldots \ldots \ldots \ldots \ldots \ldots$ & 0,735 & NS & 0,724 & 0,645 & $*$ & 0,695 \\
\hline $50 \mathrm{~kg}$-abattage $\ldots \ldots \ldots \ldots \ldots$ & 0,816 & NS & 0,778 & 0,745 & NS & 0,708 \\
\hline $27 \mathrm{~kg}$-abattage $\ldots \ldots \ldots \ldots \ldots$ & 0,779 & NS & 0,754 & 0,708 & NS & 0,703 \\
\hline \multicolumn{7}{|l|}{$\begin{array}{l}\text { Indice de consommation (kg ali- } \\
\text { ment } / \mathrm{kg} \text { gain) } \ldots \ldots \ldots \ldots \ldots \ldots\end{array}$} \\
\hline $27-50 \mathrm{~kg} \ldots \ldots \ldots$ & 2,66 & $* *$ & 2,99 & 3,04 & NS & 2,88 \\
\hline $50 \mathrm{~kg}$-abattage $\ldots \ldots \ldots \ldots \ldots$ & 3,37 & $* * *$ & 3,77 & 3,40 & ** & 3,96 \\
\hline $27 \mathrm{~kg}$-abattage $\ldots$ & 3,09 & **** & 3,49 & 3,29 & * & 3,61 \\
\hline
\end{tabular}

Signification des différences entre entiers et castrats.

NS : Non significatif. * $P<0,05$. ** $P<0,01$. *** $P<0,001$. 
TABLEAU 2

Composition corporelle

\begin{tabular}{|c|c|c|c|c|c|c|}
\hline & \multicolumn{3}{|c|}{ Première expérience } & \multicolumn{3}{|c|}{ Deuxième expérience } \\
\hline & Entiers & & Castrats & Entiers & & Castrats \\
\hline \multicolumn{7}{|l|}{$\begin{array}{l}\text { Epaisseur de lard avec peau }(\mathrm{mm}) \\
\text { au niveau: }\end{array}$} \\
\hline - du rein................... & 27,5 & *** & 35,9 & 28,3 & $* * *$ & 40,7 \\
\hline - du dos. & 23,7 & *** & 29,1 & 24,3 & $* * *$ & 31,8 \\
\hline - du cou. & 43,5 & $* * *$ & 47,7 & 45,4 & $* * *$ & 50,7 \\
\hline Densité du rein ................ & 1,039 & *** & 1,027 & 1,041 & *** & 1,025 \\
\hline Surface du muscle long dorsal $\left(\mathrm{cm}^{2}\right)$. & 30,4 & $* * *$ & 27,0 & 33,4 & $* * *$ & 29,5 \\
\hline Longe/bardière ............... & 2,18 & $* * *$ & 1,60 & 2,13 & $* * *$ & 1,49 \\
\hline Muscles/graisses $\left({ }^{1}\right) \ldots$ & 1,61 & $* * *$ & 1,13 & 1,57 & $* * *$ & 1,04 \\
\hline
\end{tabular}
(1976).

(1) Calculé à partir du rapport Longe/Bardière à l'aide de l'équation établie par Desmoulin ef al.

Signification des différences entre entiers et castrats.

NS : Non significatif. ${ }^{* *} \mathrm{P}<0,01{ }^{* * *} \mathrm{P}<0,001$.

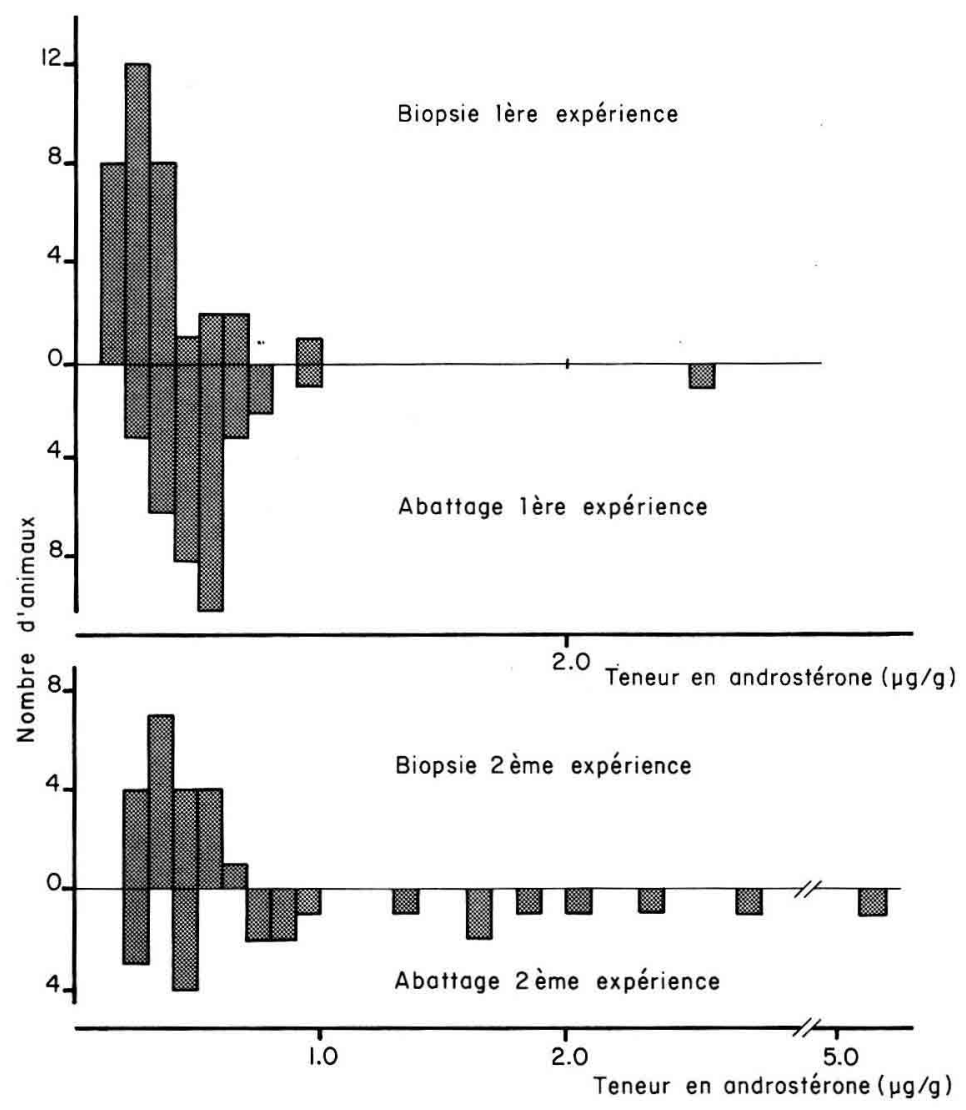

FIG. 1. - Répartition des porcs selon lo teneur en ondrosténone des graisses. 
ment selon le type sexuel. En effet les castrats ayant une consommation journalière d'aliment plus élevée de 9 à 10 p. 100, compensent ainsi leur moins bonne efficaciłé alimentaire (Indice de consommation acçu de 10 à 13 p. 100). Par ailleurs le rapport, Longe/Bardière permet d'estimer les rendements tissulaires des carcasses selon les références CEE (Desmoulin, 1978) : les carcasses des mâles entiers entrent dans la catégorie 45 à 50 p. 100 de muscles alors que celles des castrats ont moins de 40 p. $\uparrow 00$ de muscles.

2. Evolution de la teneur en androsténone entre $80 \mathrm{~kg}$ et l'abattage. - Pour les 34 porcs mâles de la première expérience, la teneur en androsténone s'élève en moyenne à $0,31 \mu \mathrm{g} / \mathrm{g}$ au moment de la biopsie à $78 \mathrm{~kg}$ de poids vif et 147 jours d'âge et à $0,55 \mu \mathrm{g} / \mathrm{g}$ au moment de l'abattage à $95 \mathrm{~kg}$ ef 167 jours. Aux deux stades la variabilité est très élevée (fig. 1). L'évolution individuelle de la concentration èn stéroïde permet de distinguer deux types d'animaux :

- pour 11 d'entre eux (32 p. 100 de l'effectif) aucune différence significative n'est mise en évidence entre les 2 stades de 78 et $95 \mathrm{~kg}$;

- pour les 23 autres (68 p. 100), la concentration en stéroïde du tissu adipeux aug; mente de façon significative de 0,28 à $0,62 \mu \mathrm{g} / \mathrm{g}$.

Dans le cas des 20 porcs mâles de la seconde expérience, la teneur en androsténone moyenne est de $0,41 \mu \mathrm{g} / \mathrm{g}$ au moment de la biopsie à $80 \mathrm{~kg}$ de poids vif et 154 jours d'âge et de $1,27 \mu \mathrm{g} / \mathrm{g}$ au moment de l'abattage à $107 \mathrm{~kg}$ et 189 jours. On peut distinguer 8 animaux (40 p. 100 de l'effectif) pour lesquels aucune différence significative n'est observée et 12 porcs (60 p. 100) pour lesquels la concentration en stéroïde augmente de 0,42 à $1,86 \mu \mathrm{g} / \mathrm{g}$.

Les teneurs en androsténone mesurées à $80 \mathrm{~kg}$ d'une part et à l'abattage d'autre part sont fortement liées $(r=+0,70 ; P<0,001)$ dans la première expérience.

TABLEAU 3

Teneurs en androsténone du tissu adipeux des porcs mâles entiers

\begin{tabular}{|c|c|c|c|c|c|c|}
\hline & \multicolumn{4}{|c|}{ Première expérience } & \multicolumn{2}{|c|}{ Deuxième expérience } \\
\hline & \multicolumn{2}{|c|}{ Individuels } & \multicolumn{2}{|c|}{ Collectifs } & \multirow[t]{2}{*}{ Individuels } & \multirow[t]{2}{*}{ Collectifs } \\
\hline & $\overline{\text { Avec }}$ 早 & Sans $q$ & Avec $q$ & Sans $q$ & & \\
\hline Nombre d'animaux $\ldots$. & 9 & 9 & 7 & 9 & 11 & 9 \\
\hline $\begin{array}{l}\text { Teneurs mesurées à la } \\
\text { biopsie }\end{array}$ & & & & & & \\
\hline $\bar{x}(\mu g / g) \quad \ldots \ldots \ldots \ldots \ldots$ & 0,30 & 0,36 & 0,26 & 0,31 & 0,37 & 0,45 \\
\hline $\begin{array}{c}\text { Coefficient de variation (p. } \\
100) \ldots \ldots \ldots \ldots \ldots \ldots \ldots\end{array}$ & 82 & 34 & 65 & 49 & 23 & 29 \\
\hline $\begin{array}{l}\text { Teneurs mesurées à l 'a- } \\
\text { battage }\end{array}$ & & & & & & \\
\hline $\bar{x}(\mu g / g) \quad \ldots \ldots \ldots \ldots$. & 0,70 & 0,48 & 0,50 & 0,51 & 0,83 & 1,82 \\
\hline $\begin{array}{l}\text { Coefficient de variation (p. } \\
100) \ldots \ldots \ldots \ldots \ldots \ldots \ldots\end{array}$ & 102 & 39 & 13 & 41 & 72 & 83 \\
\hline
\end{tabular}


En revanche, dans la seconde, le coefficient de corrélation entre ces 2 variables $n$ 'atteint pas la limite de signification ( $r=+0,30 ; N S)$.

3. Influence des conditions d'élevage. - Au moment de la biopsie, ni les conditions de logement (individuel ou en groupe) ni la présence ou l'absence de femelles n'influent sur la teneur en androsténone des graisses prélevées sur les animaux à $80 \mathrm{~kg}$ de poids vif (tabl. 3).

Lorsque les animaux sont aba,tus à un stade léger (première expérience), les concentrations en stéroïde mesurées à l'abattage ne diffèrent pas significativement selon les conditions d'élevage. Toutefois la fréquence d'animaux pour lesquels la teneur en androsténone n'augmente pas entre $80 \mathrm{~kg}$ et l'abattage est significativement plus élevée $\left(2 \hat{\imath}=5,80\left({ }^{1}\right) ; P<0,05\right)$ chez les animaux élevés en l'absence de femelles (fig. 2).

Première expérience

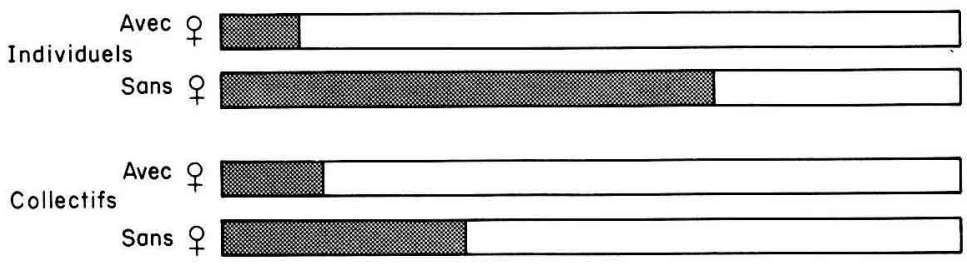

Deuxieme expérience

Individuels

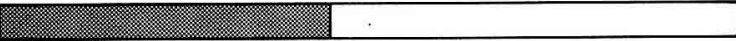

Collectifs
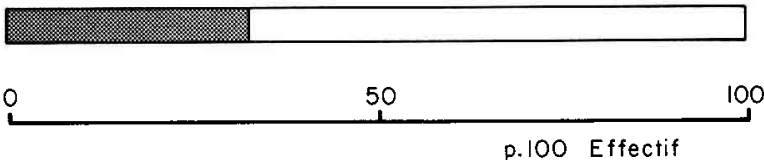

pas d'augmentation entre les 2 stades

ougmentation significative entre les 2 stades

FIG. 2. - Evolution de la teneur en androsténone entre la biopsie et l'abattage.

Lorsque les animaux sont abattus à un stade plus lourd (seconde expérience), les teneurs en androsténone sont en moyenne plus élevées $(P<0,10)$ chez les animaux élevés en loges collectives que pour les porcs en loges individuelles. Cependant, le pourcentage d'animaux pour lesquels la teneur en androsténone reste stable, ne diffère pas significativement selon les conditions de logement.

4. Relations entre la teneur en androsténone des graisses et les performances (tabl. 4). - Dans les deux expériences, la teneur en androsténone du tissu adipeux n'est pas reliée à l'âge ou au poids à l'abattage ni à aucun des critères mesurés pour caracté-

(1) Le test 2 î (Arbonnier, 1966), permet de comparer des répartitions entre différentes classes. 
riser les performances zootechniques des animaux (quantités d'aliments consommés, gain de poids vif journalier, indice de consommation).

\section{TABLEAU 4}

Coefficients de corrélation entre la teneur en androsténone à l'abattage et les performances

\begin{tabular}{|c|c|c|}
\hline & $\begin{array}{c}\text { Première expérience } \\
\text { (abattage } \\
\text { à } 95 \text { kg-166 jours) }\end{array}$ & $\begin{array}{c}\text { Deuxième expérience } \\
\text { (abattage } \\
\text { à } 107 \text { kg-189 jours) }\end{array}$ \\
\hline $\begin{array}{l}\text { Age à l'abattage } \ldots \ldots \ldots \ldots \ldots \ldots \ldots \ldots \\
\text { Poids d̀ l'abattage } \ldots \ldots \ldots \ldots \ldots \ldots \ldots \ldots \\
\text { Poids des testicules } \ldots \ldots \ldots \ldots \ldots \ldots \ldots \\
\text { Gain de poids vif } \ldots \ldots \ldots \ldots \ldots \ldots \ldots \ldots \\
\left.\text { Quantité d'aliment consommée }{ }^{1}\right) \ldots \ldots \ldots \\
\text { Indice de consommation }(1) \ldots \ldots \ldots \ldots \ldots \\
\text { Longueur de la carcasse } \ldots \ldots \ldots \ldots \ldots \ldots \\
\text { Epaisseur de lard : }\end{array}$ & $\begin{array}{r}-0,20 \\
-0,19 \\
-0,05 \\
0,15 \\
0,28 \\
0,13 \\
0,06\end{array}$ & $\begin{array}{c}-0,00 \\
0,19 \\
-0,02 \\
-0,26 \\
0,23 \\
0,33 \\
0,52 * *\end{array}$ \\
\hline 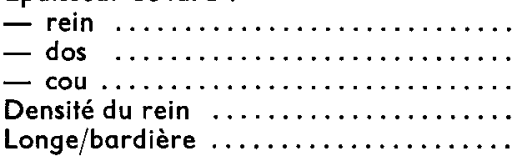 & $\begin{array}{r}0,17 \\
-0,03 \\
0,02 \\
-0,07 \\
-0,16\end{array}$ & $\begin{array}{l}-0,29 \\
-0,44 * \\
-0,32 \\
0,53 * * \\
0,36\end{array}$ \\
\hline
\end{tabular}

(1) Données établies pour les porcs en loges individuelles seulement.

$* P<0,10$. ** $P<0,05$.

Les relations entre la concentration en stéroïde et les critères de composition corporelle sont faibles et non significatives lorsque les animaux sont abattus à un stade léger (première expérience).

Dans la seconde en revanche (abattage à $107 \mathrm{~kg}$ ), la teneur en androsténone est reliée positivement aux critères de développement musculaire (densité du rein) ef négativement aux critères d'adiposité (épaisseurs de lard dorsal).

\section{Discussion.}

1. Evolution de la teneur en androsténone. - Selon les premières observations de Claus (1975) chez le jeune porc mâle entier, la teneur en androsténone du tissu adipeux est d'abord stable à un niveau faible $(<0,3 \mu \mathrm{g} / \mathrm{g})$ puis augmente après 140 jours d'âge. Les résultats de cette éfude permettent d'apporter quelques nuances à ce schéma général d'évolution. La grande variabilité de la teneur en androsténone observée au moment de la biopsie à $80 \mathrm{~kg}$ de poids vif et 150 jours d'âge indique que, conformément aux observations d'Andresen (1976), l'âge auquel la concentration en ce stéroïde commence à augmenter est extrêmement variable selon les individus. Ainsi, le moment où la teneur en androsténone commence à augmenter est postérieur au stade usuel d'abattage chez un tiers environ des animaux considérés dans cette étude. Les porcs présentant des teneurs faibles à l'abattage appartiennent dans leur grande majorité à cette catégorie. 
A l'inverse une augmentation significative de la concentration en stéroïde esł observée chez tous les animaux présentant des teneurs élevées à l'abattage.

Le niveau de production festiculaire d'androsténone est étroitement lié à l'intensité de la synthèse de testostérone (Groth et Claus, 1977). Il est donc intéressant d'établir un parallèle entre nos résultats ef l'évolution de la testostéronémie décrite par Meusy-Dessolle (1975) chez de jeunes porcs mâles de même race. Les stades d'abattage considérés dans cetfe étude encadrent l'âge de 180 jours pour lequel une augmentation brutale de la testostérone plasmatique est décrite par cet auteur. Lorsque les animaux sont abattus à 167 jours (première expérience), les teneurs en androsténone sont en moyenne faibles. En revanche, dans la seconde expérience (abattage à 189 jours) ces valeurs sont beaucoup plus élevées.

La concentration en androsténone du tissu adipeux augmentant avec l'âge, on peut penser qu'une détermination de la concentration en ce stéroïde effectuée sur une biopsie de tissu gras à $80 \mathrm{~kg}$ de poids vif, pourraił permettre de prévoir la teneur en androsténone à l'abattage. Cet objectif esł partiellement atteint (coefficiẹnt de détermination $r^{2}=0,49$ ) lorsque ces 2 stades ne sont séparés quẹ par un court intervalle (20 jours et $17 \mathrm{~kg}$ de gain de poids au cours de la première expérience). II n'en va pas de même dans le cas de la seconde expérience où les intervalles sont plus longs (35 jours ef $27 \mathrm{~kg}$ ).

2. Influence des conditions d'élevage. - L'influence des conditions d'élevage sur l'évolution de la feneur en androsténone en fin d'engraissement ou sur les niveaux de concentration en ce stéroïde à l'abattage est en moyenne difficile à évaluer en raison de la variabilité intra lot qui reste considérable. La maîtrise des conditions d'élevage ne permet donc pas de contrôler totalement les niveaux de stockage d'androsténione. Toutefois, l'engraissement de jeunes porcs mâles entiers en groupe ou en présence de femelles semble favoriser le stockage de ce stéroïde. Les résultats des tests olfactifs réalisés lors du chauffage du tissu adipeux dorsal et de la cuisson des viandes des animaux de ces deux expériences permettent de dégager les mêmes tendances (Desmoulin et Bonneau, résultats non publiés). Ces observations rejoignent les conclusions de Walker (1978), selon lesquelles l'élevage de jeunes porcs mâles entiers en présence de jeunes truies peut augmenter la fréquence des défauts d'odeur des viandes des jeunes mâles.

3. Relations avec les performances. - Bien que les limites des stades d'abattage considérés dans chacune des deux expériences soient assez étroites (coefficients de variation de 3 p. 100 pour le poids et de 7 p. 100 pour l'âge), la variabilité de la teneur en androsténone du tissu adipeux est très élevée. Dans ces conditions, aucune relation significative n'est mise en évidence entre la teneur en androsténone et le poids ou l'âge à l'abattage. Ainsi alors que pour une population de jeunes porcs mâles entiers la concentration en stéroïde augmente en moyenne avec le poids ou l'âge, pour un animal considéré isolément le poids ou l'âge ne permettent pas de préjuger de la teneur en androsténone du fissu adipeux de cet individu.

Lorsqu'on abat de jeunes animaux (95 kg, 167 jours), les teneurs en androsténone sont faibles et on peut penser que ces animaux ont été abattus avant de pouvoir exprimer leurs potentialités de synthèse de ce stéroïde. Dans ces conditions la teneur en androsténone est indépendante des performances, conformément aux résultats 
obłenus précédemment (Bonneau et Desmoulin, 1979). Lorsque les porcs sont abattus à un stade plus tardif, les teneurs en stéroïde à l'abattage sont en moyenne plus élevées. On observe alors que les mâles dont les carcasses ont les pourcentages de muscles les plus importants ont tendance à présenter les teneurs en stéroïdes les plus élevées.

La teneur en androsténone étant un caractère moyennement héritable, il conviendrait donc d'éfudier dans quelle mesure il est possible de sélectionner des animaux présentant à poids ou à âge fixe des concentrations faibles en stéroïde sans préjudice sur le niveau des performances zoolechniques.

Reçu en novembre 1979. Accepté en mars 1980.

\section{Références}

ANDRESEN O., 1976. Concentrations of fat and plasma $5 \alpha$-androstenone and plasma testosterone in boars selected for rate of body weight gain and thickness of back fat during growth sexual maturation and after mating. J. Reprod. Fert., 48, 51-59.

ARBONNIER P., 1966. L'analyse de l'information : aperçu théorique et application à la loi multinominale. Ann. Sci. for., 23, 950-1020.

BONNEAU M., DESMOULIN B., 1979. Teneurs en androsténone des graisses de jeunes porcs mâles issus de croisement de type "Camborough 》. Ann. Zootech., 28, 185-190.

CLAUS R., 1974. Dosage radioimmunologique du 5- $\alpha$-androst-16-ene-3-one, steroïde responsable de l'odeur de verrat, dans le tissu adipeux des porcs. C. R. Acad. Sci. Paris, Série D, 278, $299-$ 302.

CLAUS R., 1975. Messung des Ebergeruchstoffes im Fett von Schweinen mittels eines Radioimmunotests 1 Mitteilung : Geruchsdepotbildung in Abhängigheit vom alter. Z. Tierzüchtg. Züchtungsbiol., 92, 118-126.

DESMOULIN B., 1970. La détermination de la densité corporelle : Application à l'étude des états d'obésité chez le porc, Journées Rech. Porcine en France, 2, 171-186, I. N. R. A.-I. T. P. éd., Paris.

DESMOULIN B., 1978. Etude sur la composition corporelle du porc : applications scientifiques ou techniques. Journées Rech. Porcine en France, 10, 211-234, I. N. R. A.-I. T. P. éd., Paris.

DESMOULIN B., GRANDSART P., TASSENCOURT L., 1976. Les critères d'appréciation de la composition anatomique de la carcasse de porc et des pièces de découpe : principes généraux ef difficultés de classification, Journées Rech. Porcine en France, 8, 89-98, I. N. R. A.-I. T. P. éd., Paris.

GROTH W., CLAUS R., 1977. Beziehungen zwischen den Konzentrationen von Testosteron und dem Ebergeruchstoff 5- $\alpha$-androst-16-ene-3-one im Blutt bzw. Fettgewebe und histometrischen Befunden im Hoden vom Schwein. Zbl. Vet. Med. A., 24, 103-121.

HEMSWORTH P. H., BEILHARZ R. G., GALLOWAY D. B., 1977a. Influence of social conditions during rearing on the sexual behaviour of the domestic boar. Anim. Prod., 24, 245-251.

HEMSWORTH P. H., WINFIELD C. G., BEILHARZ R. G., GALLOWAY D. B., 1977b. Influence of social conditions post puberty on the sexual behaviour of the domestic male pig. Anim. Prod., 25, 305-309.

HEMSWORTH P. H., FINDLAY J. K., BEILHARZ R. G., 1978. The importance of physical contact with other pigs during rearing on the sexual behaviour of the male domestic pig. Anim. Prod., 27, 201-207.

MEUSY-DESSOLLE N., 1975. Variations quantitatives de la testostérone plasmatique chez le porc mâle, de la naissance à l'âge adulte. C. R. Acad. Sci. Paris, Sér. D, 281, 1875-1878.

PATTERSON R. L. S., $1968 . \quad 5$ - $\alpha$-androst-16-ene-3-one ; compound responsible for taint in boar fat. J. Sci. Fd. Agric., 19, 31-38.

THOMAS H. R., KATTESH H. G., KNIGHT J. W., GWAZDAUSKAS F. C., MEACHAM T. N., KORNEGAY E. T., 1979. Effects of housing and rearing on age at puberty and libido in boars. Anim. Prod., 28, 231-234.

WALKER N., 1978. Meat production from boars. Agric. Res. Inst. North. Irel., Hillsborough, 51th. Annu. Rep., 25-28. 